\title{
Le acidosi tubulari
}

\author{
Marco Quaglia, Guido Merlotti, Vincenzo Cantaluppi
}

Dipartimento di Medicina Traslazionale, Unità di Nefrologia e Trapianto, A.O.U. "Maggiore della Carità" di Novara, Università del Piemonte Orientale, Novara

\begin{abstract}
Renal tubular acidosis
Renal tubular acidosis (RTA) refers to a group of disorders of multiple etiology characterised by a hyperchloremic, normal anion-gap metabolic acidosis caused by different defects affecting several mechanisms of urinary acidification: an impaired secretion of protons by collecting duct in distal hypokalemic RTA (type 1), impaired resorption of bicarbonate in proximal RTA (type 2), a mixed proximal and distal defect in RTA type 3 and an impaired ammoniagenesis due to an absolute or functional hypoaldosteronism in distal hyperkalemic RTA (type 4). Etiology is heterogeneous and ranges from primitive genetic forms to secondary RTA within a multi-system disorder such as autoimmune disease, paraproteinemia and calcium-phosphate disorders. Drug-induced RTA has been increasingly reported over the last decade due to widespread use of some molecules (e.g. antiviral and oncologic agents, inhibitors of renin-angiotensin system). The diagnosis of RTA is based on assessment of serum and urine anion-gap, whereas differential diagnosis between RTA forms requires the analysis of urine $\mathrm{pH}$ or osmolality and of serum and urine electrolytes. An acidification test should be performed to confirm the diagnosis. Clinical setting can also provide important clues in orienting towards a type of RTA: nephrolithiasis and nephrocalcinosis are associated with type 1 RTA, ostemalacia and osteoporosis are more frequent in type 2 RTA, osteopetrosis is typically linked to type 3 RTA and hyperkalemia is the predominant manifestation of type 4 RTA. An increased awareness of RTA is needed among clinical Nephrologists to recognise it and prevent potentially severe complications.
\end{abstract}

Keywords: Aldosterone, Hypokalemia, Hyperkalemia, Nephrolithiasis, Nefrocalcinosis, Renal Tubular Acidosis

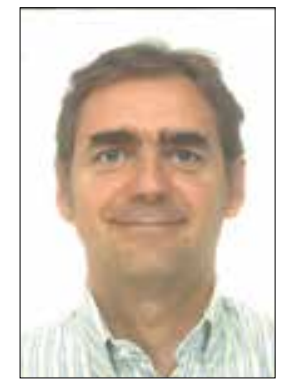

Marco Quaglia

\section{Definizione e classificazione}

Le acidosi tubulari (renal tubular acidosis, RTA) sono condizioni accomunate da una ridotta escrezione tubulare netta di $\mathrm{H}^{+}$a causa di varie alterazioni della funzione tubulare, spesso in assenza di compromissione del filtrato glomerulare (1-3).

Le RTA vengono classificate in base al meccanismo fisiopatologico (Tab. I) in:

Accepted: April 16, 2016

Published online: July 11, 2016

Indirizzo per la corrispondenza:

Dr. Marco Quaglia

Dipartimento di Medicina Traslazionale

Unità di Nefrologia e Trapianto

Università del Piemonte Orientale

Via Solaroli 17

28100 Novara

marco.quaglia@gmail.com

- $\quad$ RTA tipo 1 (distale ipokaliemica): caratterizzata da una ridotta secrezione di $\mathrm{H}^{+}$nel dotto collettore (DC), per cui il $\mathrm{pH}$ urinario, che fisiologicamente può essere ridotto fino a 4.5-5 in questi segmenti, rimane al di sopra di 5.3 nonostante la presenza di un'acidosi metabolica.

- RTA tipo 2 (prossimale): caratterizzata da un ridotto riassorbimento di $\mathrm{HCO}^{-}$a livello del tubulo contorto prossimale (TCP), da un pH urinario adeguato all'acidosi $(<5.3)$ solo quando la bicarbonatemia si riduce al di sotto di una soglia critica e inadeguatamente elevato (>5.3) in caso contrario.

- RTA tipo 3 (mista prossimale e distale): caratterizzata da anomalie dell'anidrasi carbonica di tipo 2 (CA 2) che condizionano sia un ridotto riassorbimento di $\mathrm{HCO}^{-}$che un difetto di ammoniogenesi.

- RTA tipo 4 (distale iperkaliemica): caratterizzata da un ipoaldosteronismo (assoluto o funzionale) che condiziona un difetto nell'acidificazione distale attraverso un'inibizione dell'ammoniogenesi e della secrezione $\mathrm{di}^{+} \mathrm{da}$ parte del DC. II pH urinario è variabile.

L'acidosi metabolica tipica della RTA è caratterizzata da un anion gap (AG) normale (acidosi metabolica ipercloremica), 
TABELLA I - Caratteristiche fisiopatologiche e cliniche delle diverse forme di RTA

\begin{tabular}{llll}
\hline & Tipo $\mathbf{1}$ (distale) & Tipo 2 (prossimale) & Tipo 4 \\
\hline Meccanismo fisiopatologico & Ridotta acidificazione distale & $\begin{array}{l}\text { Ridotto riassorbimento di } \\
\text { bicarbonato prossimale }\end{array}$ & Ipoaldosteronismo \\
pH urinario durante l'acidosi & $>5.3$ & $\begin{array}{l}\text { Variabile a seconda della } \\
\text { bicarbonatemia }\end{array}$ & Spesso $<5.3$ \\
Bicarbonatemia & Può essere $<10 \mathrm{mEq} / \mathrm{L}$ & $16-18 \mathrm{mEq} / \mathrm{L}$ & $16-22 \mathrm{mEq} / \mathrm{L}$ \\
Frazione escreta di $\mathrm{HCO}_{3}$ & $<3 \%$ & $>15 \%$ & $<3 \%$ \\
Potassiemia & Ridotta & Normale o ridotta & Aumentata \\
Citraturia & Bassa & Alta & Bassa \\
Complicanze non elettrolitiche & Nefrolitiasi e nefrocalcinosi & $\begin{array}{l}\text { Osteomalacia e rachitismo; } \\
\text { ritardo di crescita }\end{array}$ & Nessuna \\
\hline
\end{tabular}

a differenza dell'acidosi metabolica dell'insufficienza renale cronica (normocloremica, con anion gap aumentato) (cfr. paragrafo Inquadramento diagnostico) (1-3).

La RTA di tipo IV è già stata oggetto di una Rassegna pubblicata su questa Rivista (4), per cui verrà trattata in questa sede più concisamente, mentre ci focalizzeremo sulle RTA di tipo 1 e $2(5,6)$.

\section{Fisiopatologia ed eziologia}

I principali trasportatori che regolano l'equilibrio acidobase a livello del TCP, delle cellule principali (CP) e delle cellule intercalate di tipo $\alpha(\alpha \mathrm{Cl})$ del DC sono descritti rispettivamente nelle Figure 1 e 2; il meccanismo dell'ammoniogenesi è descritto nella Figura 3.

\section{RTA di TIPO 1}

Sono stati dimostrati 4 meccanismi fisiopatologici che sottintendono l'alterata secrezione di $\mathrm{H}^{+}$nei dotti collettori $(1,2$, 5, 7) (Fig. 2):

a) deficit di secrezione di $\mathrm{H}^{+}$intrinseco, secondario a deficit dell' $\mathrm{H}^{+}$-ATPasi dell' $\alpha \mathrm{Cl}$ del DC, tipico della forma "classica" (secretory defect);

b) deficit di secrezione di $\mathrm{H}^{+}$secondario a incapacità di generare un gradiente elettrico transepiteliale con negatività endoluminale a causa di un ridotto riassorbimento di $\mathrm{Na}^{+}$da parte del canale epiteliale di $\mathrm{Na}^{+}(\mathrm{ENaC})$ della $\mathrm{CP}$ del DC (voltage-dependent defect);

c) deficit di secrezione di $\mathrm{H}^{+}$secondario a un'aumentata retrodiffusione di $\mathrm{H}^{+}$normalmente secreti, a causa di un'alterata permeabilità del tubulo distale (gradient defect);

d) deficit di secrezione di $\mathrm{H}^{+}$secondario a ridotto trasporto di $\mathrm{NH}_{4}{ }^{+}$dal TCP all'interstizio della midollare (low-buffer type).

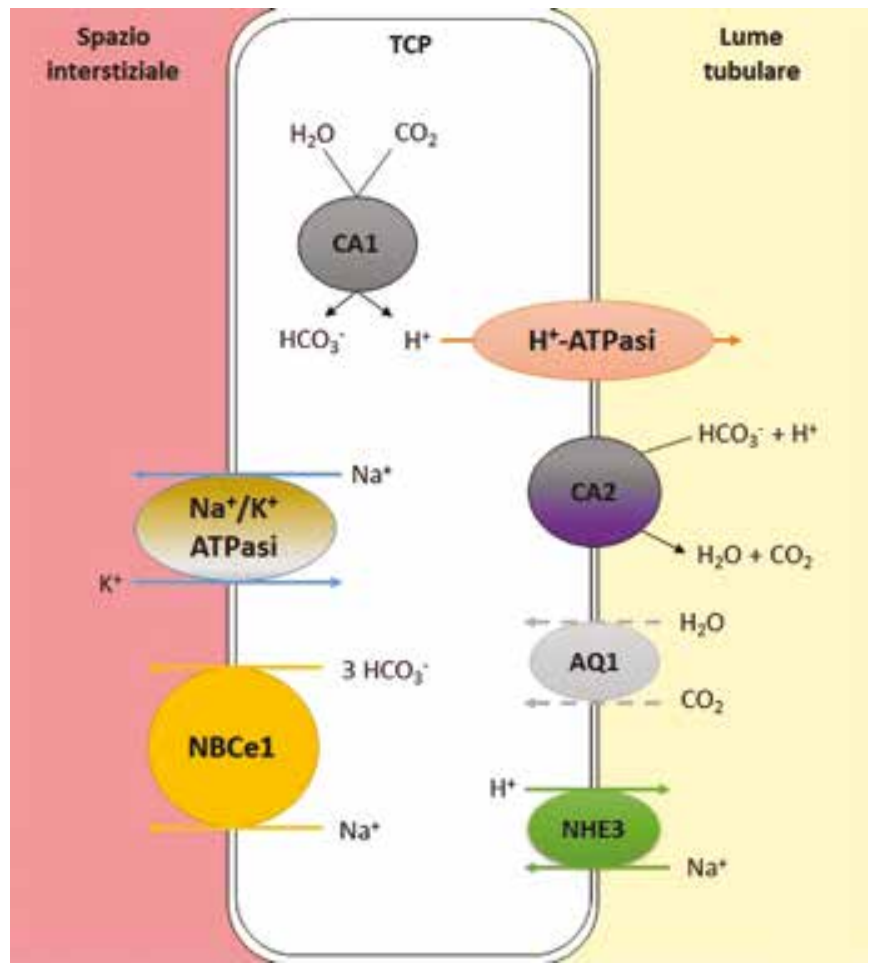

Fig. 1 - Meccanismo di acidificazione urinaria prossimale. TCP: tubulo contorto prossimale; AQ1: acquaporina 1. CA 2: anidrasi carbonica intracellulare di tipo 2. CA 4: anidrasi carbonica di membrana di tipo 4. NHE3: antiporto $\mathrm{Na}^{+} / \mathrm{H}^{+}$di tipo 3; NBCe1: simporto $\mathrm{Na}^{+} / \mathrm{HCO}_{3}^{-}$.

a) II deficit di secrezione di $\mathrm{H}^{+}$intrinseco rappresenta il principale e peculiare deficit di acidificazione della RTA di tipo 1 ed è stato ricondotto ad anomalie di vari trasportatori a livello dell' $\alpha \mathrm{Cl}$ del DC (Fig. 2), che sono state descritte, per esempio, nella malattia di Sjögren (7-9). Alcuni pazienti hanno un difetto isolato della $\mathrm{H}^{+} / \mathrm{K}^{+}$ATPasi, che determina, oltre alla mancata secrezione di $\mathrm{H}^{+}$, anche un deficit del riassorbimento di $\mathrm{K}^{+}$con conseguente 


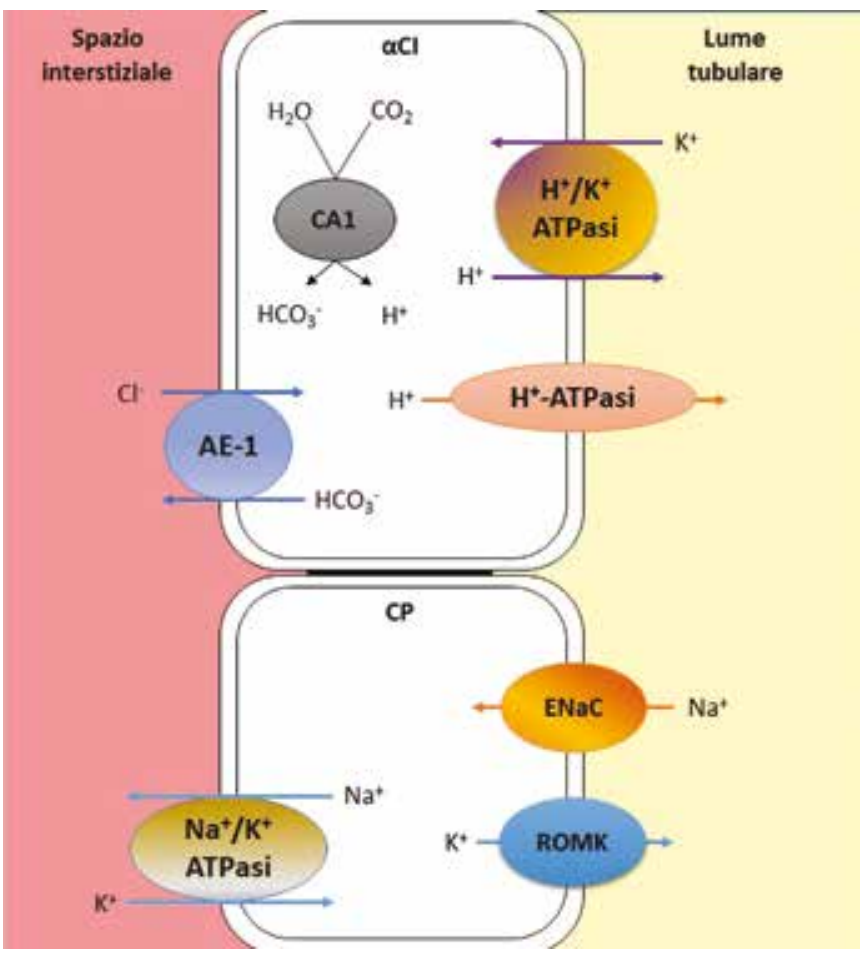

Fig. 2 - Meccanismo di acidificazione urinaria distale. $\alpha \mathrm{Cl}$ : cellula intercalata di tipo $\alpha$. CP: cellula principale. AE-1: antiporto $\mathrm{HCO}_{3}^{-1} \mathrm{Cl}^{-}$; ENaC: canale epiteliale per il $\mathrm{Na}^{+}$; ROMK: canale epiteliale per il $\mathrm{K}^{+}$.

ipokaliemia. In altri è presente un difetto isolato della $\mathrm{H}^{+}$-ATPasi vacuolare, anch'esso associato a un effetto di wasting del $\mathrm{K}^{+}$oppure a un difetto nello scambiatore di anioni basolaterale (basolateral anion exchanger, BAE) che, impedendo l'eliminazione di $\mathrm{HCO}_{3}^{-}$attraverso la membrana basolaterale, alcalinizza la cellula tubulare inibendo la secrezione di $\mathrm{H}^{+}(7)$. Sono anche state caratterizzate forme genetiche di RTA di tipo 1 causate da mutazioni a carico di questi medesimi enzimi, a trasmissione sia autosomica dominante che recessiva (Tab. II).

b) II deficit di secrezione di $\mathrm{H}^{+}$secondario a incapacità a generare o a mantenere un gradiente elettrico transepiteliale è causato da una riduzione del riassorbimento $\mathrm{di}^{\mathrm{Na}^{+}}$a livello della corticale per un deficit della $\mathrm{Na}^{+} / \mathrm{K}^{+}$-ATPasi (Fig. 2) ed è stato descritto nella forme di uropatia ostruttiva, nell'anemia falciforme e nel trapianto renale. La riduzione dell'elettronegatività endoluminale che ne consegue (voltage defect) determina una riduzione della secrezione non solo di $\mathrm{H}^{+}$, ma anche di $\mathrm{K}^{+}$per cui questa forma costituisce la cosiddetta "variante iperkaliemica" della RTA di tipo 1, che, nella sua forma classica (a), è invece tipicamente associata a ipokaliemia. È da sottolineare che anche un'importante riduzione della volemia efficace, tale da comportare una riduzione del carico distale di $\mathrm{Na}^{+}$può indurre reversibilmente, in modo "funzionale", questa variante di RTA distale (sindrome nefrosica, sindrome epato-renale) (1-3).
TABELLA II - Principali cause di RTA di tipo 1 secondaria

\author{
Disordini autoimmuni \\ - Malattia di Sjogren \\ - Artrite reumatoide \\ - LES \\ - Cirrosi biliare primitiva \\ - Ipergammaglobulinemia (p. es., cirrosi, paraproteinemia)
}

Farmaci e tossine

- Amfotericina B

- Litio

- Abuso di analgesici

- Toluene

Disordini del metabolismo calcio-fosforico con nefrocalcinosi

- Iperparatiroidismo primitivo

- Ipervitaminosi D

- Ipercalciuria idiopatica

Nefropatia tubulo-interstiziale cronica

- Uropatia ostruttiva

- Trapianto renale

- Anemia falciforme

Importante deplezione di volume e condizioni di iponatriuria

Disordini genetici

- RTA autosomica dominante: difetto di AE1

- RTA autosomica recessiva: difetto nella subunità A4 dell'H+'-ATPasi

- RTA autosomica recessiva con sordità neurosensoriale progressiva: difetto nella subunità B1 dell' $\mathrm{H}^{+}$-ATPasi

c) Una ridotta concentrazione endoluminale di $\mathrm{H}+$ secondaria ad aumentata retrodiffusione di $\mathrm{H}+$ normalmente secreti, a causa di un'alterata permeabilità del tubulo distale ("gradient defect"), determina una riduzione del "trapping" dello ione ammonio $\mathrm{NH}_{4}^{+}$nel lume del dotto collettore e la persistenza di $\mathrm{NH}_{3}$, che puo' diffondere liberamente (1-3) (Fig. 3).

d) Un ridotto "recycling" di NH4+ dal tubulo contorto prossimale all'interstizio della midollare - a causa di una nefropatia tubulo-interstiziale cronica secondaria a ipokaliemia o di una nefrocalcinosi - può aggiungere un'altra componente di danno a quella descritta in c), determinando una carenza del buffer costituito dal sistema $\mathrm{NH}_{3} / \mathrm{NH}_{4}{ }^{+}$("lowbuffer").

I meccanismi b) e d) possono essere presenti nella RTA di tipo 1, mentre sono predominanti nell'altra forma di RTA distale, la RTA tipo 4, nella quale sono entrambi causati dall'ipoaldosteronismo e dall'iperkaliemia (4).

L'eziologia della RTA distale comprende sia forme primitive, idiopatiche o ereditarie, sia forme secondarie a disordini 
sistemici (Tab. II). La malattia di Sjögren $(8,9)$, vari disordini autoimmuni (10) e le ipergammaglobulinemie sono le condizioni più spesso associate a questo disordine. Molto più rare sono le forme di RTA ereditarie che sono state descritte nella malattia di Marfan e di Ehlers-Danlos, nella malattia di Wilson e nell'ipercalciuria familiare. Le forme primitive genetiche a trasmissione autosomica recessiva sono state associate a mutazioni dei geni codificanti per le subunità A4 e B1 della $\mathrm{H}^{+}$-ATPasi e per lo scambiatore anionico basolaterale AE1, mentre la forma autosomica dominante è stata associata solo a mutazioni a carico di AE1 (11-13) (Fig. 2).

\section{RTA di TIPO 2}

Il meccanismo fisiopatologico fondamentale della RTA di tipo 2 è una riduzione della soglia del riassorbimento di $\mathrm{HCO}_{3}$ - da parte del TCP (normalmente pari a 26-28 mEq/L) per cui, in presenza di una bicarbonatemia normale, la quantità di bicarbonato filtrata dal glomerulo eccede la capacità di riassorbimento del TCP (che riassorbe fisiologicamente circa il $90 \%$ dell' $\mathrm{HCO}_{3}$ - filtrato) e anche quella dell'ansa di Henle e del tubulo distale, determinando una perdita di bicarbonato nelle urine (con aumento della $\mathrm{FE}$ dell' $\mathrm{HCO}_{3}{ }^{-}$spesso oltre il $15-20 \%$ ) con consensuale riduzione della bicarbonatemia. Quando la quantità di $\mathrm{HCO}_{3}^{-}$filtrata si riduce a un livello corrispondente alla soglia riassorbitiva del tubulo, la perdita di $\mathrm{HCO}_{3}^{-}$cessa e si realizza un nuovo "steady state" di bicarbonatemia a un livello inferiore rispetto a quello fisiologico. È da sottolineare come la capacità riassorbitiva dell' $\mathrm{HCO}_{3}{ }^{-} \mathrm{da}$ parte del nefrone distale sia invece integra, contribuendo ad autolimitare il disordine (anche in presenza di un blocco tota-
TABELLA III - Principali cause di RTA di tipo 2 acquisita

Paraproteinemia

- Mieloma multiplo

- Tubulopatia prossimale da catene leggere in LCDD

- Amiloidosi

Iperparatiroidismo primario e secondario

Deficit di vitamina D

Farmaci e tossine

- Inibitori dell'anidrasi carbonica (Acetazolamide, Topiramato)

- Inibitori della trascriptasi inversa nucleotidici (Tenofovir, Adefovir) e nucleosidici (Lamivudina)

- Antineoplastici (Ifosfamide, Cisplatino, Oxaliplatino)

- Anticonvulsivanti (Acido valproico)

- Antibiotici (Aminoglicosidi) e antivirali (Cidofovir)

- Metalli pesanti (piombo, mercurio, cadmio, rame)

- Acido aristolocico

Nefropatia tubulo-interstiziale cronica

- Trapianto renale

- Malattia cistica della midollare

le del riassorbimento tubulare prossimale la bicarbonatemia non scende al di sotto di $10 \mathrm{mEq} / \mathrm{L})(1,2,6,13)$.

L'ipokaliemia, generalmente presente in questa forma, è secondaria sia a un'attivazione del sistema renina-angiotensina a causa della contrazione della volemia causata dalla perdita di $\mathrm{HCO}_{3}^{-}$(con conseguente aumento del carico distale di $\mathrm{Na}^{+}$, iperaldosteronismo secondario e aumentata

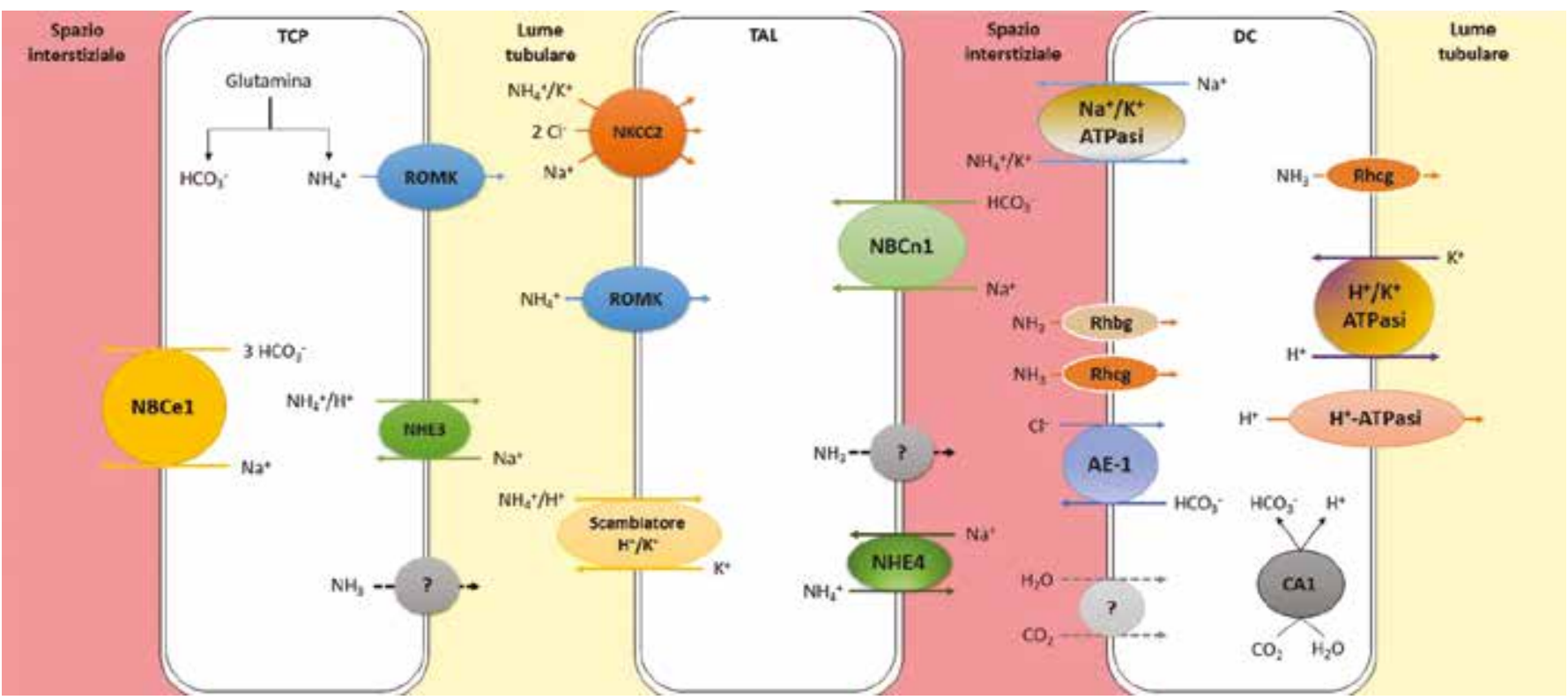

Fig. 3 - Meccanismo dell'ammoniogenesi. NKCC2: simporto $\mathrm{Na}+\mathrm{K}^{+} / \mathrm{Cl}^{-}$. $\mathrm{NBCn1}$ : simporto $\mathrm{Na}^{+} / \mathrm{HCO}_{3}$. $\mathrm{NHE}$ : antiporto $\mathrm{Na}^{+} / \mathrm{H}^{+}$di tipo 4 . Rhbg: glicoproteina Rhesus di tipo B; Rhcg: glicoproteina Rhesus di tipo C. 
secrezione di $\mathrm{K}^{+}$) sia a un wasting di $\mathrm{K}^{+}$.

La RTA di tipo 2 può essere isolata oppure, molto più spesso, può realizzarsi nel contesto di un disordine più ampio della funzione tubulare prossimale, la sindrome di Fanconi, caratterizzata da un ridotto riassorbimento non solo di $\mathrm{HCO}_{3}^{-}$, ma anche di glucosio (glicosuria normoglicemica), fosfato (con ipofosforemia), acido urico (con ipouricemia), aminoacidi e proteine a basso peso molecolare (14).

La presenza di una sindrome di Fanconi deve indurre a ricercare una possibile condizione associata, acquisita (Tab. III) o congenita. Tra le prime, quella più frequente nell'adulto è la presenza di una mieloma multiplo con produzione di catene leggere libere monoclonali, che supera la capacità riassorbitiva del TCP, causandone una sofferenza, spesso in presenza di una proteinuria di Bence-Jones (15). Tra le forme congenite è da ricordare l'associazione della sindrome di Fanconi con la cistinosi, la tirosinemia, la galattosemia, la malattia di Wilson, la malattia di Lowe e la malattia di Dent $(1,2,13,14)$.

\section{RTA di TIPO 3}

Questa forma è causata da anomalie genetiche di CA 2. II difetto di acidificazione è caratterizzato da wasting di bicarbonato, incapacità di ridurre il $\mathrm{pH}$ urinario $<5.5$ e ridotta escrezione di $\mathrm{NH}^{+}(13,16)$.

\section{RTA di TIPO 4}

Questa forma di RTA distale è causata da un difetto di acidificazione causato da un ipoaldosteronismo assoluto (riduzione dei livelli circolanti di ormone) o funzionale ("resistenza" all'aldosterone per disfunzione del DC), che determina un'inibizione dell'ammoniogenesi (Fig. 3) e una riduzione del riassorbimento di $\mathrm{Na}^{+}$, con conseguente "voltage defect", e della secrezione di $\mathrm{K}^{+}$e $\mathrm{H}^{+}$(1-3) da parte rispettivamente di CP e $\alpha \mathrm{Cl}$ (Fig. 2). L'iperkaliemia, a sua volta, inibisce marcatamente la disponibilità di $\mathrm{NH}_{4}^{+}$a livello del DC (buffer defect), interferendo a vari livelli con il processo di ammoniogenesi (ridotta sintesi a livello del TCP e ridotto riassorbimento di $\mathrm{NH}_{4}^{+}$a livello del TAL dell'ansa di Henle, per competizione tra $\mathrm{NH}^{+} \mathrm{e}$ $\mathrm{K}^{+}$) (Fig. 3). L'AG urinario è positivo, indicando un'inadeguata escrezione urinaria di $\mathrm{NH}_{4}^{+}$. Nei pazienti nei quali la RTA di tipo 4 è causata da una riduzione dei livelli circolanti di aldosterone, il pH urinario è tipicamente $<5.3$ (prevalente difetto nell'ammoniogenesi), mentre in quelli in cui è presente un danno strutturale del DC (prevalente difetto nella secrezione di $\left.\mathrm{H}^{+}\right)$il $\mathrm{pH}$ è $>5.3(1,4,17,18)$.

La RTA di tipo 4 è stata trattata in dettaglio in una Rassegna pubblicata su questa Rivista (4).

\section{Inquadramento diagnostico}

Ai fini di un corretto inquadramento è essenziale il calcolo dell'AG sierico (sAG), dell'AG urinario ( $U A G$ ) ed eventualmen- te dell'osmolarità urinaria (uOsm), le cui rispettive formule sono riportate di seguito (1-3):

$$
\begin{aligned}
& \text { AG: }\left[\mathrm{Na}^{+}-\left(\mathrm{Cl}^{-}+\mathrm{HCO}_{3}^{-}\right)\right] \\
& \text {uAG: }\left(\mathrm{Na}^{+}+\mathrm{K}^{+}\right)-\mathrm{Cl}^{-} \\
& \text {uOsm: } 2 \times\left[\mathrm{Na}^{+}+\mathrm{K}^{+}\right]+\text {Azoturia/6+ Glucosio/18 }
\end{aligned}
$$

\section{a) Valutazione dell’AG sierico}

Tutte le forme di RTA sono caratterizzate da un'acidosi metabolica ipercloremica con AG sierico normale. Questa caratteristica le differenzia dall'acidosi metabolica normocloremica con AG aumentato, che si riscontra in corso di insufficienza renale cronica avanzata (GFR $>20 \mathrm{~mL} / \mathrm{min}$ ), acidosi lattica e chetoacidosi diabetica, da malnutrizione e alcolica (1-3).

\section{b) valutazione dell'AG urinario (uag) o dell'osmolarità urinaria (uosm)}

La positività dell'uAG è tipica soprattutto della forme di RTA distali, caratterizzate da una ridotta escrezione di $\mathrm{NH}_{4}{ }^{+} \mathrm{Cl}$ e, quindi, da una cloruria inadeguatamente bassa, con conseguente positivizzazione dell'uAG (il quale invece, in condizioni fisiologiche, si negativizza in presenza di acidosi metabolica). Nella RTA prossimale, I'uAG può essere negativo, ma in minor misura rispetto a quanto fisiologicamente atteso. La presenza di un AG francamente negativo orienta, quindi, verso una forma di acidosi da perdita gastroenterica di $\mathrm{HCO}_{3}^{-}$(diarrea persistente, presenza di uretero-sigmoidostomia o drenaggi pancreatici/biliari), testimoniando la presenza di una normale acidificazione urinaria distale.

Quando il pH urinario supera 6.5, l'accuratezza dell'uAG nel misurare l'escrezione di $\mathrm{NH}_{4}^{+}$si riduce, per cui è indicato l'impiego dell'uOsm. L'escrezione di $\mathrm{NH}_{4}^{+}$è considerata adeguata nel contesto di un'acidosi se il valore di uOsm è superiore a $100 \mathrm{mOsm} / \mathrm{Kg}(1-3)$.

\section{c) Diagnosi differenziale tra le forme di RTA}

Una volta diagnosticata la presenza di una RTA, la diagnosi differenziale tra le diverse forme si basa su elementi laboratoristici e sul contesto clinico (Tab. I).

Un primo step consiste nel valutare la funzione tubulare prossimale. La diagnosi di RTA di tipo 2 si basa sulla dimostrazione di un'elevata FE del bicarbonato $>15 \%$ ( $>5 \%$ nelle forme meno severe), mentre il $\mathrm{pH}$ urinario può ridursi a $<5.5$ se la bicarbonatemia è inferiore alla soglia renale di riassorbimento ed essere invece inadeguatamente $>5.5$ in caso contrario (Tab. I). Deve essere valutata, inoltre, la presenza di altri difetti del tubulo prossimale: la presenza di glicosuria normoglicemica e di un'aumentata FE di fosforo, acido urico e aminoacidi, oltre alla presenza di una proteinuria tubulare, configura RTA di tipo 2 nel contesto di una sindrome di Fanconi $(1,3)$.

Una volta esclusa una RTA prossimale, è necessario ese- 
guire una diagnosi differenziale tra le due forme di acidosi distale. La presenza di un $\mathrm{pH}$ urinario persistentemente $>5.3$ durante l'acidosi orienta verso la diagnosi di RTA di tipo 1 (ridotta escrezione di $\mathrm{H}^{+}$a causa di un danno del dotto collettore), soprattutto in presenza di normo-ipokaliemia, mentre la presenza di un $\mathrm{pH}<5.3$ è compatibile con una RTA di tipo 4 (ridotta disponibilità di $\mathrm{NH}_{4}{ }^{+}$a livello distale per ipoaldosteronismo). Occorre, tuttavia, tenere in considerazione che una stimolazione dell'ammoniogenesi determina una riduzione della concentrazione urinaria di $\mathrm{H}^{+} \mathrm{e}$, quindi, un aumento del $\mathrm{pH}$ che non corrisponde necessariamente a un difetto di acidificazione; per contro nella RTA di tipo 4, caratterizzata principalmente da un deficit di ammoniogenesi, il pH urinario può essere $<5.3$ (si riduce l'acidità titolabile escreta come $\mathrm{NH}_{4}^{+}$e, quindi, la concentrazione di $\mathrm{H}^{+}$ liberi aumenta). La presenza di un'infezione delle vie urinarie con organismi produttori di ureasi e di un'iposodiuria può, inoltre, determinare un $\mathrm{pH}$ alcalino che non esprime alcun difetto di acidificazione (3). Al fine di determinare la reale capacità di acidificazione delle urine è quindi opportuno ricorrere a un test di acidificazione. II test con furosemide (1 mg/kg per via orale, max: $40 \mathrm{mg}$ ) e fludrocortisone $(0.025 \mathrm{mg} / \mathrm{Kg}$, max: $0.1 \mathrm{mg})(\mathrm{FF})(19)$, meglio tollerato rispetto al test tradizionale con assunzione orale di cloruro di ammonio, si basa sulla somministrazione simultanea del diuretico dell'ansa per aumentare il carico di $\mathrm{Na}^{+}$distale e del mineralcorticoide per stimolare i meccanismi di acidificazione a livello del dotto collettore; qualora il pH urinario, misurato ogni ora per 4-6 ore dopo l'assunzione dei due farmaci, sia $<5.5$ in una qualsiasi rilevazione, il meccanismo di acidificazione distale è considerato normale. Al contrario, la persistenza del $\mathrm{pH}>5.5$ orienta verso una diagnosi di RTA di tipo 1 (deficit di secrezione di $\mathrm{H}^{+}$secondario a danno del DC). Se, invece, la kaliemia è aumentata e il pH urinario scende $<5.5$, la risposta è compatibile con una RTA di tipo IV (ipoaldosteronismo "funzionale" con inibizione dell'ammoniogenesi, in assenza di danno anatomico tubulo-interstiziale) (17).

Altri elementi utili per la diagnosi differenziale tra RTA distali sono la kaliemia, spesso ridotta nella RTA di tipo 1 (almeno nella forma "classica") e sempre aumentata nella RTA di tipo 4, e l'ipercalciuria (calciuria/creatininuria $>0.2$ ), tipica della RTA di tipo 1 (mentre l'ipocitraturia è presente in entrambe le forme distali) (Tab. I).

Infine, anche l'anamnesi e il contesto clinico possono orientare la diagnosi differenziale. La presenza di nefrolitiasi/ nefrocalcinosi è tipica della RTA tipo 1; quadri di osteomalacia/osteoporosi, anche se conseguenza dell'acidosi metabolica di per sé, sono molto più frequenti nella RTA tipo 2 per la coesistenza di altri fattori favorenti (ipofosforemia e deficit di vitamina D). Inoltre, alcune patologie sistemiche si associano tipicamente a certi tipi di RTA (p. es., malattia di Sjogren e RTA di tipo 1; mieloma multiplo e RTA tipo 2), così come numerosi farmaci (Tabb. I e II) $(1-3,20)$.
La RTA di tipo 4 è particolarmente frequente nell'anziano diabetico, spesso in presenza di un certo grado di malattia renale cronica (chronic kidney disease, CKD) e con il concorso di farmaci favorenti $(4,17)$. Tuttavia può verificarsi più raramente in altri contesti, come il lupus eritematoso sistemico (LES) (21) e la gravidanza (22).

L'esame obiettivo deve includere l'analisi del fundus oculi per escludere cristalli di cistina nella cistinosi (sindrome di Fanconi) e l'esame audiometrico per escludere sordità (forma autosomica dominante di RTA distale) (3).

\section{Manifestazioni cliniche}

\section{RTA di TIPO 1 (distale)}

I pazienti con RTA di tipo 1 sono spesso asintomatici oppure sviluppano sintomi legati all'acidosi metabolica spesso severa o all'ipokaliemia (astenia, poliuria, polidipsia, stipsi e paralisi muscolare). Nei bambini possono verificarsi un ritardo nella crescita e deformità ossee, qualora l'acidosi non venga corretta tempestivamente (1). La forma genetica autosomica recessiva esordisce nei primi mesi di vita e si complica con nefrocalcinosi e sordità precoce o tardiva. La forma autosomica dominante è, invece, meno severa, esordisce nell'adolescenza o in età adulta e si associa a una nefrocalcinosi in modo incostante (11-13).

In generale, la nefrolitiasi e la nefrocalcinosi sono comuni (23-25). Un recente studio condotto su pazienti con nefrolitiasi calcica recidivante ha dimostrato che la prevalenza di RTA distale completa e incompleta era rispettivamente pari al $7 \%$ e al $13,7 \%$ se si impiegava il test di acidificazione con furosemide e fludrocortisone per la diagnosi (19). Diversi importanti fattori predisponenti alla nefrolitiasi (soprattutto fosfato di calcio) si realizzano nella RTA di tipo $1(1,5)$ :

- ipercalciuria e iperfosfaturia, secondarie a demineralizzazione ossea indotta dall'acidosi metabolica e ridotto riassorbimento tubulare del calcio a causa del $\mathrm{pH}$ endoluminale alcalino;

ipocitraturia, secondaria sia all'acidosi (aumentata conversione del citrato a bicarbonato nella cellula tubulare, con conseguente aumentato riassorbimento tubulare del citrato) che all'ipokaliemia cronica;

- $\quad$ pH urinario persistentemente alcalino (che promuove la precipitazione di fosfato di calcio).

In alcune famiglie, l'ipercalciuria precede l'acidosi metaboli$\mathrm{ca}$, suggerendo che la RTA sia, in questi casi, la conseguenza di un primitivo disordine del bilancio calcio-fosforico (26); tuttavia, nella maggior parte dei casi, l'ipercalciuria è direttamente proporzionale all'acidosi e ne rappresenta una complicanza.

La RTA di tipo 1 ha una prevalenza di circa il 5\% nella malattia di Sjogren, della quale può rappresentare la manifestazione di esordio $(9,10)$, ed è stata descritta in associazione con il LES e con l'artrite reumatoide (8). 


\section{RTA di TIPO 2 (prossimale)}

Il rachitismo nel bambino e quadri di osteomalacia e osteoporosi nell'adulto si verificano in circa il $20 \%$ dei casi di RTA di tipo $2(27,28)$. L'eziologia di queste manifestazioni è multifattoriale: oltre all'acidosi metabolica, vi contribuiscono il wasting di fosfato con conseguente ipofosforemia e il deficit di vitamina D (essendo il TCP una sede fondamentale della sintesi di calcitriolo). La nefrolitiasi e la nefrocalcinosi non sono associate a questa forma, con l'eccezione della RTA secondaria a topiramato, un farmaco anti-comiziale che inibisce la CA e determina anche ipercalciuria e ipocitraturia (20).

La RTA isolata autosomica recessiva è associata a un severo ritardo di crescita, anomalie oculari (glaucoma, cataratta, keratopatia a banda) e ritardo mentale, mentre la forma autosomica dominante è caratterizzata da un lieve ritardo mentale e da una ridotta massa ossea. Entrambe richiedono una terapia alcalinizzante permanente $(13,14)$. La RTA isolata acquisita è causata dagli inibitori della CA.

Spesso la RTA prossimale si verifica nel contesto di un disordine più ampio della funzione tubulare prossimale, la sindrome di Fanconi, causato da una patologia sistemica (paraproteinemia) o dall'assunzione di farmaci (13) (Tab. II).

\section{RTA di TIPO 3}

Questa forma può essere ereditaria e associarsi a osteopetrosi, calcificazioni cerebrali e ritardo mentale oppure essere un fenomeno transitorio nel bambino (16).

\section{RTA di TIPO 4}

Il quadro clinico è dominato dalle manifestazioni dell'iperkaliemia, sproporzionata rispetto alla funzione renale (spesso è presente una CKD moderata, a differenza degli altri tipi di RTA, nei quali la funzione renale è normale) e preminente rispetto all'acidosi metabolica. Il paziente è, in genere, anziano e affetto da diabete mellito in circa il $50 \%$ dei casi (ipoaldosteronismo iporeninemico) e, spesso, in terapia con associazioni di farmaci inibitori dell'asse renina-angiotensina-aldosterone. Solitamente, è presente un evento emodinamico favorente, tale da ridurre la volemia efficace e, quindi, il carico di $\mathrm{Na}^{+}$al DC (scompenso cardiaco, ipovolemia), creando le condizioni per una ridotta escrezione di $\mathrm{K}^{+}$(voltage defect) $(4,17,18)$.

\section{Terapia}

\section{RTA di TIPO 1}

La terapia della RTA di tipo 1 si basa sulla somministrazione a lungo termine di bicarbonato di $\mathrm{Na}^{+}(1-2 \mathrm{mmol} / \mathrm{Kg} /$ die) e supplementi di $\mathrm{K}^{+}$. Nel paziente con nefrolitiasi recidivante la correzione dell'acidosi aumenta l'escrezione uri- naria di citrato, contribuendo a rallentare la formazione di nuovi calcoli $(1,2,5)$.

\section{RTA di TIPO 2}

In questo contesto, la terapia è più problematica, in quanto la somministrazione di bicarbonato di $\mathrm{Na}^{+}$tende ad aumentare la perdita urinaria di bicarbonati impedendo la correzione completa dell'acidosi. Inoltre, l'aumento del carico distale di $\mathrm{Na}^{+}$, in concorso con un iperaldosteronismo secondario alla cronica deplezione di volume, può determinare un wasting di $\mathrm{K}^{+}$con peggioramento dell'ipokaliemia. È, quindi, indicata la supplementazione di basi sotto forma di citrato di $\mathrm{K}^{+}$. Nei bambini, il trattamento deve essere aggressivo, al fine di minimizzare l'impatto dell'acidosi sulla crescita e richiede, in genere, dosi elevate di basi $(5-15 \mathrm{mmol} / \mathrm{Kg} / \mathrm{die})$. Nell'adulto asintomatico, invece, si tende a eseguire la somministrazione di bicarbonato di $\mathrm{Na}^{+}$se la bicarbonatemia è inferiore a 18 $\mathrm{mmol} / \mathrm{L}$, anche per la complessità di assumere dosi elevate di basi (circa $700-1000 \mathrm{mmol} /$ die) $(1,2,6,14)$.

\section{RTA di TIPO 3}

Non esiste una terapia specifica per questa rara forma, nella quale sono impiegabili sia il bicarbonato di $\mathrm{Na}+$ che il citrato di $\mathrm{K}+$ essendo presente un disordine misto sia prossimale che distale $(12,13)$.

\section{RTA di TIPO 4}

Il provvedimento fondamentale è la riduzione della kaliemia, che spesso è sufficiente di per sé a correggere contemporaneamente anche l'acidosi metabolica, in quanto elimina l'effetto inibitorio dell'iperkaliemia sull'ammoniogenesi e, quindi, ripristina il "buffer" $\mathrm{di} \mathrm{NH}_{4}^{+}$a livello distale $(4,5,7)$. Un'adeguata idratazione in caso di ipovolemia e una terapia diuretica nel paziente iperteso o con scompenso cardiaco possono essere utili per aumentare il carico distale di $\mathrm{Na}^{+}$e, quindi, stimolare la secrezione di $\mathrm{K}^{+} \mathrm{e} \mathrm{H}^{+}$a questo livello (4). Nei pazienti con ipoaldosteronismo, può essere indicata la somministrazione di fludrocortisone $(0.1 \mathrm{mg} / \mathrm{die})$. La terapia con bicarbonato di sodio può essere impiegata per facilitare la riduzione della kaliemia, purché non sia presente un sovraccarico di volume. È fondamentale eliminare (almeno temporaneamente) eventuali farmaci inibenti l'asse renina-angiotensina-aldosterone (ACE-inibitori, sartani, anti-aldosteronici, ma anche $\beta$-bloccanti e FANS) valutando attentamente il rapporto rischio/beneficio di queste terapie nel singolo paziente, soprattutto qualora comprendano associazioni di farmaci (p. es., beta-bloccante, ACE-inibitore e spironolattone in un paziente diabetico con scompenso cardiaco) $(17,18)$. Per una trattazione più approfondita di questi aspetti farmacologici si rimanda alla Rassegna giù pubblicata su questa Rivista (4). 


\section{Conclusioni}

Le RTA sono un gruppo di disordini caratterizzati da un'acidosi metabolica ipercloremica (AG normale) secondaria a vari difetti nei meccanismi dell'acidificazione tubulare a livello prossimale (deficit del riassorbimento dei bicarbonati) o distale (deficit della secrezione $\mathrm{di}^{+}$e/o deficit di buffer per ridotta ammoniogenesi). Mentre alcune di queste forme sono geneticamente determinate a esordio infantile e relativamente rare, le RTA acquisite rappresentano un aspetto di rilevante importanza per il Nefrologo clinico, in considerazione dell'impiego sempre più esteso di farmaci favorenti (p. es., farmaci oncologici e anti-virali e farmaci interferenti con l'asse-reninaangiotensina-aldosterone) e dell'elevata prevalenza di alcuni disordini sistemici associati (soprattutto gammopatie monoclonali, malattie autoimmuni per le RTA di tipo 1 e 2; diabete mellito per RTA di tipo 4). Una maggiore consapevolezza di queste tubulopatie può condurre a una diagnosi più precoce di alcune patologie sistemiche, delle quali rappresentano, talora, la prima manifestazione, e alla prevenzione di importanti complicanze: dalla nefrolitiasi/nefrocalcinosi a disionie severe (29).

\section{Disclosures}

Financial support: No financial support was received for this submission.

Conflict of interest: The authors have no conflict of interest.

\section{Bibliografia}

1. Floege J, Johnson RJ, Feehally J. Metabolic Acidosis. In: Comprehensive Clinical Nephrology. 2011;155-161. Ed. Elsevier Saunders.

2. Rodriguez SJ. Renal tubular acidosis: the clinical entity. J Am Soc Nephrol. 2002;13(8):2160-70.

3. Sharma S, Gupta A, Saxena S. Comprehensive clinical approach to renal tubular acidosis. Clin Exp Nephrol. 2015;19(4):556-61.

4. Quaglia M, Merlotti G, Izzo C, Stratta P. L'Acidosi tubulare di tipo IV: una nefropatia emergente. GTND. 2014;26(4):329-37.

5. Kurtz I. Molecular mechanisms and regulation of urinary acidification. Compr Physiol. 2014;4(4):1737-74.

6. Nakamura M, Shirai A, Yamazaki O, et al. Roles of renal proximal tubule transport in acid/base balance and blood pressure regulation. Biomed Res Int. 2014;2014:504808.

7. Roy A, Al-bataineh MM, Pastor-Soler NM. Collecting duct intercalated cell function and regulation. Clin J Am Soc Nephrol. 2015;10(2):305-24.

8. Both T, Zietse R, Hoorn EJ, et al. Everything you need to know about distal renal tubular acidosis in autoimmune disease. Rheumatol Int. 2014;34(8):1037-45.

9. Evans R, Zdebik A, Ciurtin C, Walsh SB. Renal involvement in primary Sjögren's syndrome. Rheumatology (Oxford). 2015;54 (9):1541-8.

10. Both T, Hoorn EJ, Zietse $R$ et al. Prevalence of distal renal tubular acidosis in primary Sjögren's syndrome. Rheumatology
(Oxford). 2015;54(5):933-9.

11. Escobar L, Mejía N, Gil H, Santos F. Distal renal tubular acidosis: a hereditary disease with an inadequate urinary $\mathrm{H}^{+}$excretion. Nefrologia. 2013;33(3):289-96.

12. Batlle D, Haque SK. Genetic causes and mechanisms of distal renal tubular acidosis. Nephrol Dial Transplant. 2012;27(10): 3691-704.

13. Alper SL. Familial renal tubular acidosis. J Nephrol. 2010;23(Suppl. 16):S57-76.

14. Haque SK, Ariceta G, Batlle D. Proximal renal tubular acidosis: a not so rare disorder of multiple etiologies. Nephrol Dial Transplant. 2012;27(12):4273-87.

15. Stokes MB, Valeri AM, Herlitz L, et al. Light Chain Proximal Tubulopathy: Clinical and Pathologic Characteristics in the Modern Treatment Era. J Am Soc Nephrol. 2015. pii: ASN.2015020185. [Epub ahead of print].

16. Sh Ali AA, Al-Mashta SA. Cerebral calcification, osteopetrosis and renal tubular acidosis: is it carbonic anhydrase-Il deficiency? Saudi J Kidney Dis Transpl. 2013;24(3):561-5.

17. Sousa AG, Cabral JV, El-Feghaly WB, de Sousa LS, Nunes AB. Hyporeninemic hypoaldosteronism and diabetes mellitus: Pathophysiology assumptions, clinical aspects and implications for management. World J Diabetes. 2016;7(5):101-11.

18. Palmer BF, Clegg DJ. Electrolyte and Acid-Base Disturbances in Patients with Diabetes Mellitus. N Engl J Med. 2015;373(6): 548-59.

19. Shavit L, Chen L, Ahmed F, et al. Selective screening for distal renal tubular acidosis in recurrent kidney stone formers: initial experience and comparison of the simultaneous furosemide and fludrocortisone test with the short ammonium chloride test. Nephrol Dial Transplant. 2016. pii: gfv423. [Epub ahead of print].

20. Hall AM, Bass P, Unwin RJ. Drug-induced renal Fanconi syndrome. QJM. 2014;107(4):261-9.

21. Jakes AD, Baynes K, Nelson-Piercy C. Renal tubular acidosis type 4 in pregnancy. BMJ Case Rep. 2016;2016.

22. Sánchez-Marcos C, Hoffman V, Prieto-González S, et al. Renal tubular acidosis type IV as a complication of lupus nephritis. Lupus. 2016;25(3):307-9.

23. Tasic V, Gucev Z. Nephrolithiasis and Nephrocalcinosis in Children - Metabolic and Genetic Factors. Pediatr Endocrinol Rev. 2015;13(1):468-76.

24. Fonseka CL, Galappaththi SR, Karunarathna JD, et al. A case of multiple myeloma presenting as a distal renal tubular acidosis with extensive bilateral nephrolithiasis. BMC Hematol. 2016;16:8.

25. El Ati Z, Fatma LB, Boulahya G, et al. Osteomalacia complicating renal tubular acidosis in association with Sjogren's syndrome. Saudi J Kidney Dis Transpl. 2014;25(5):1072-7.

26. Lo TE, Tan IT. Distal renal tubular acidosis in primary hyperparathyroidism. BMJ Case Rep. 2015;2015. pii: bcr2014208120. doi: 10.1136/bcr-2014-208120.

27. Miller PD. Unrecognized and unappreciated secondary causes of osteoporosis. Endocrinol Metab Clin North Am. 2012;41(3): 613-28.

28. Taylor HC, Elbadawy EH. Renal tubular acidosis type 2 with Fanconi's syndrome, osteomalacia, osteoporosis, and secondary hyperaldosteronism in an adult consequent to vitamin $\mathrm{D}$ and calcium deficiency: effect of vitamin $D$ and calcium citrate therapy. Endocr Pract. 2006;12(5):559-67.

29. Golembiewska E, Ciechanowski K. Renal tubular acidosisunderrated problem? Acta Biochim Pol. 2012;59(2):213-7. 